\title{
Development of a Universal Scale for Validating the Process of Sanitization of Equipment, Materials and Surfaces in the Animal Facility
}

\author{
A.S. Chernov, A.N. Minakov, E.V. Malyavina, A.S. Elfimov', G.B. Telegin
}

10.18805/IJAR.B-1387

\begin{abstract}
Background: Monitoring of sanitization of cage equipment is an essential function of any laboratory animal facilities, seeking to ensure the animal health and welfare. The purpose of the current study was to develop universal scale for monitoring sanitization through detection of residual ATP for the most effective process of sanitizing components of rodents micro- and macro-environment. Methods: Sterile pens and swabs for the HY-LiTE® Luminometer Instrument were used to measure ATP concentration (RLU) on the cleaned surface samples. We have examined the elements of the microenvironment (rodent and rabbit cage, cage wire meshes, feed separating pieces, water bottles with tips, houses, tunnels, IVC cage frames, cage tops and cage wire meshes). The assessed swab area on each of the surfaces was $10 \times 10 \mathrm{~cm}$.

Result: We observed, that combined washing (cleaned manually and with automatic universal washers with detergent) gives the lowest RLU values. Monitoring of the quality of sanitization of equipment and surfacescan be carried out without the use of microbiological tests. Use of pre-washing allows increasing the service life of caging equipment. We have developed an assessment scale to monitor sanitizing, which can be recommended to scientific and breeding animal facilities for monitoring sanitization cage equipments.

Key words: Animal facility ATP-test, Cages, Luminometer, Rodents, Sanitization.

Abbreviation: AAALAC- The Association for Assessment and Accreditation of Laboratory Animal Care; ATP- Adenosine triphosphate; HACCP- Hazard analysis and critical control points; RLU- Relative light units; SPF- Specified pathogen free; ABF- Animal breeding facility, RAS- Russian Academy of Science; RODAC - Replicate organism direct agar contact.
\end{abstract}

\section{INTRODUCTION}

Monitoring of the sanitization of surfaces, materials and equipment used for laboratory animals housing and breeding is an essential technological process impacting the animal welfare (Guide for the Care and Use of Laboratory Animals 2011). As a rule, in the SPF-animal facilities autoclaving is performed at the final stage of equipment and material sanitization to ensure their sterility. However, some animal facilities employ only washing and disinfection processes not followed by sterilization. As a result, a poor-quality sanitization may lead to animal infections and, in turn, affect the results of experimental research.

According to the AAALAC requirements, the effectiveness of both manual and automatic sanitizing methods should be evaluated on a regular basis (Guide for the Care and Use of Laboratory Animals 2011). To assess the cleanliness of the processed materials we propose a microbiological test and a system for detecting the presence of organic debris with bioluminescence method based on measurements of adenosine triphosphate (ATP). Bacteria and other types of microbial contamination of surfaces can also be detected by using contact agar plates (RODAC) (Bruch and Smith, 1968, Hart et al., 1973, Hacek et al., 2000).

However, sometimes it is not possible to detect biological contamination using this test (Vilar et al., 2008). Fragments of live and dead cells serve as a nutritive substrate facilitating rapid microbial growth (Turner et al.,
Animal Breeding Facility, Branch of the Shemyakin and Ovchinnikov Institute of Bioorganic Chemistry of the Russian Academy of Sciences, Pushchino-142290, Russia.

"Merck" LLC, subsidiary of Merck KGaA in Russia, Moscow 117198, Russia.

Corresponding Author: G.B. Telegin, Animal Breeding Facility, Branch of the Shemyakin and Ovchinnikov Institute of Bioorganic Chemistry of the Russian Academy of Sciences, Pushchino142290, Russia. Email: telegin@bibch.ru

How to cite this article: Chernov, A.S., Minakov, A.N., Malyavina, E.V., Elfimov, A.S. and Telegi, G.B. (2022). Development of a Universal Scale for Validating the Process of Sanitization of Equipment, Materials and Surfaces in the Animal Facility. Indian Journal of Animal Research. DOI: 10.18805/IJAR.B-1387.

Submitted: 18-05-2021 Accepted: 18-12-2021 Online: 16-01-2022

2010 and Srinivasan et al., 2019). In contrast with the microbiological techniques, the ATP measurement can be used as a rapid test to determine the level of contamination and to implement preventive measures without waiting for the active bacterial growth (Whiteley et al., 2016). In view of its high mobility and excellent reliability, the luminometer is considered as an operator friendly analytical instrument that helps to avoid the use of complex microbiological equipment. Also, it does not yield false positive results. Compared to RODAC, the ATP-based measurements test 
is a cost effective, easy-to-perform and high-reproducibility method which does not require highly skilled personnel (Ednie et al., 1998).

Strict standards of detecting residual ATP as an indicator of surface contamination were developed for the food industry in accordance with the HACCP requirements (Handbook of Hygiene Control in the Food Industry, 2005). ATP testing is used commonly in the aviation industry for monitoring the condition of aviation fuel when it comes to microbial presence (Gaylarde et al., 1999 and Rauch et al., 2006). In addition multiple studies were carried out to monitor the effectiveness of hospital cleaning practices (Boyce et al., 2009, Sherlock et al., 2009, Mulvey et al., 2011). The ATP bioluminescence could be considered a practical as well as a method to assess hospital hygiene, performing better than visual inspection (namely, Bacharach scale, bassoumeter and glossmeter), if properly adopted (Nante et al., 2017).

Nevertheless, no uniform criteria are currently available to make a quantitative evaluation of ATP-contamination of different equipment and materials employed in animal care and use facilities. Researchers suggest $1000 \mathrm{RLU}$ as a cutoff value in laboratory mouse and rat housing facilities (Schondelmeyer et al., 2006 and Horn et al., 2012). Other studies highlighted that a range of 100-250 RLU is acceptable for mouse caging equipment (Saren et al., 2016 and Smith et al., 2018). Technology-related differences between the agricultural sector, chemical industry, medicine and the veterinary field urge to develop individual standards for assessing ATP concentrations.

The aim of this study was to propose an assessment scale for monitoring sanitization through detection of residual ATP and to develop the most effective process of sanitizing components of animal micro- and macro-environment in the Animal Breeding Facility, Branch of Shemyakin and Ovchinnikov Institute of Bioorganic Chemistry, Russian Academy of Sciences ABF BIBCh, RAS.

\section{MATERIALS AND METHODS What is the "ABF BIBCh RAS"?}

Laboratory animals in the ABF BIBCh, RAS comprise inbred and outbred laboratory mice, rats, hamsters and rabbits of SPF-category, according to recommendation of the Federation of European Laboratory Animal Science Associations (FELASA 2014), as well as genetically modified mice used as animal models of Alzheimer disease, Duchenne disease and amyotrophic lateral sclerosis. All species held in the ABF BIBCh, RAS are included in the Bioresource collection of experimental SPF-rodents used for fundamental, biomedical and pharmacological research.

The ABF BIBCh, RAS is focused in particular on the development of precision hemilateral glial scar of spinal cord in rats and diffuse alveolar damage of lung in mice (Telegin et al., 2021, Sharapova et al., 2021). The facility has approx. 6000 cages. Since 2004, the ABF BIBCh, RAS has been accredited by AAALAC and since 2007 has achieved the certification of the Quality Management System under the
ISO Standard 9001:2015. The ABF BIBCh, RAS is a part of the unique research unit (UNU) "Biomodel" of the Institute of Biochemistry, RAS and have the "Bioresource Collection of SPF-Laboratory Rodents for Fundamental, Biomedical and Pharmacological Studies'.

\section{Animal husbandry}

Mice, rats, hamster and rabbits in the ABF BIBCh are held in the standard conditions according to the Institutional Animal Care and Use Program and the IACUC-approved SOPs. The open cages are sanitized simultaneously with the changing of bedding material every 7-10 days and the individually ventilated cage (IVC) - every 10-14 days. Rabbit cages are changed weekly and cage waste trays with soiled bedding - every 3 days. Lignocel and Arbocel (Rettenmaier, Germany) are used as bedding materials in the cages with small rodents. The numbers of animals placed in cages is determined according to the requirements of Guide for the Care and Use of Laboratory Animals, 2011.

\section{Elements of the microenvironment}

Rodent cage frames; rabbit cage frames and waste trays; cage wire meshes; feed separating pieces; water bottles with tips; houses; tunnels, IVC cage frames,cage tops and cage wire meshes. All elements are produced by Tecniplast, Italy.

\section{Types of washing}

\section{1) Manual washing without detergents}

Sanitization with warm tap water;

\section{2) Manual washing with the use of detergents}

Sanitization by sequential immersing in detergent solutions Neodisher FLA (0.6\%) and Neodisher N (0.5\%) (Dr. Weigert, Germany) at $+30^{\circ} \mathrm{C}$ without presoaking. Final rinsing in warm tap water.

\section{3) Automatic washing in universal washers}

- 650GP series (Tecniplast, Italy) for rodent cages and cage accessories with the phase times: washing at $+65^{\circ} \mathrm{C}$, Neodisher FLA $0.1 \%$, 200 s; water drain phase $10 \mathrm{~s}$; neutralizing with Neodisher $\mathrm{N} 0.2 \%, 7 \mathrm{~s}$; washing at $+85^{\circ} \mathrm{C}$, $30 \mathrm{~s}$; drying phase $60 \mathrm{~s}$. Type of washing "class B".

- 9EW series (Tecniğlast, Italy) for water bottles and tips with the following phase times: rinsing $10 \mathrm{~s}$; washing at $+55^{\circ} \mathrm{C}$, Neodisher $\mathrm{N} 0.2 \%, 90 \mathrm{~s}$; water draining phase $5 \mathrm{~s}$; washing at $+60^{\circ} \mathrm{C}, 20 \mathrm{~s}$; draining phase $3 \mathrm{~s}$; drying phase 50 s. "Type of washing class B".

- Atlantis EVO series (Tecniplast, Italy) for rabbit cages, waste trays and racks with the following phase times: washing at $+84^{\circ} \mathrm{C}$, Neodisher $\mathrm{N} 0.2 \%, 180 \mathrm{~s}$; drain phase $15 \mathrm{~s}$; rinsing at $+86^{\circ} \mathrm{C}, 25 \mathrm{~s}$; drain phase $5 \mathrm{~s}$, thermal disinfection with clean steam at $+95^{\circ} \mathrm{C}, 300 \mathrm{~s}$; drying at $+95^{\circ} \mathrm{C}, 150 \mathrm{~s}$. "Type of washing class A".

\section{4) Automatic washing in universal washers with manual pre-washing}

cages and accessories are soaked in alkaline detergent Neodisher FLA $(0.4 \%)$ at $+30^{\circ} \mathrm{C}$ for $15 \mathrm{~min}$, cleaned 
mechanically with a sponge and then loaded in automatic washer chamber.

Wire meshes of rodent cages and rabbit cage waste trays are soaked in super-foam for touch-free washing (Active Foam Pink, Grass). Then, mechanical cleaning in tap water is done with high-pressure Karcher K5 (Italy) and, as the final step, the equipment is loaded in automatic washer.

Water bottle tips are soaked in alkaline detergent Neodisher A8 $(0.2 \%)$ at $+30^{\circ} \mathrm{C}$ for $15 \mathrm{~min}$. Then, mechanical cleaning is done in tap water coming out from the shower (9ES, Tecniplast, Italy). As the final step, the equipment is loaded in automated washer.

\section{5) Automatic washing in universal washers with manual pre-washing with autoclaving}

Washing equipment as in point 4) and autoclave sterilization. Sterilization parameters: number of vacuum cycles: 3 , sterilization temperature: $121^{\circ} \mathrm{C}$, sterilization time 0 minutes. Drying time is different for each type of material: 70 minutes.

\section{Assessment of the quality of sanitization}

The HY-LiTE ${ }^{\circledR}$ test system (Merck KGaA, Darmstadt, Germany) was used to assess the hygiene status. The total ATP (including bacterial and non-bacterial contamination caused by products and their residues) was measured for monitoring cleanliness of work surfaces. Bioluminescence test exploits the chemiluminescence properties of luciferin-luciferase reagent, which reacts with any ATP residue present on a substrate, emitting light and measuring the presence of organic matter (Shama and Malik 2013 and Dancer, 2014). Sterile pens and swabs for the HYLiTE $®$ Luminometer Instrument were used to measure ATP concentration (in RLU) on the cleaned surface samples.

We set a threshold value (green range scale) for microenvironment elements as a mean value ATP level after automated washing without prewashing. For the yellow range scale the threshold value was a mean value ATP level after manual washing with detergent. Further increase in the threshold value was attributed to the red area of the scale. All experiments to measure RLU were performed between September 2018 and March 2021.

\section{Statistical analysis}

All data RLU are presented as mean \pm SEM for twenty five independent replicates. The statistical test carried out is stated in the legend of the corresponding table, employing t-tests in comparisons between treatment. $p<0,05$ и $p<0,01$ values were considered as statistically significant. At all times, to assess the cleanliness, 550 tests of microenvironment elements were performed. The assessed swab area on each of the surfaces was $10 \times 10 \mathrm{~cm}$.

\section{RESULTS AND DISCUSSION}

The results (Table 1 ) demonstrate that the mean RLU values were $2772.4 \pm 556.7$ for the microenvironment components cleaned manually with warm tap water (without adding detergent). The use of caging equipment with such contamination level is unacceptable. The results of manual washing with added detergents demonstrated a 16-fold decrease in RLU values, to $158.6 \pm 36.2$, compared to manual washing without detergent These levels suggest that the caging equipment is well-cleaned and can be used (Schondelmeyer et al., 2006 and Horn et al., 2012). The obtained results demonstrate that the ATP test will be helpful for conducting in-house control of the adequate performance of equipment sanitation process. In this context, if an employee "forgets" to add a cleaning detergent for disinfection, this failure will become obvious as soon as the test is carried out. This fact has been proven by our experimental findings.

Both methods of manual washing are characterized by a high variability (more than five-fold) of RLU values for different microenvironment elements, because the cleaning procedures are not standardized, e.g. due to the impact of human factor. It would be possible to avoid this variability, if all manual washing operations are performed by the same employee, but this option is not realistic. As an alternative, automated washing could be used.

The concentration of ATP in the microenvironment elements cleaned in automated washers was measured (Table 1). As shown in the Table, in those microenvironment elements that were cleaned in automatic universal washers with detergent without pre-washing the RLU values to 135.4

Table 1: Results of RLU-measurements of the samples taken from the surfaces of microenvironment elements manually and automated washed.

\begin{tabular}{|c|c|c|c|}
\hline Type of washing & & Mean, RLU & \pm SEM \\
\hline \multirow[t]{2}{*}{ Manual washing } & without detergent & 2772.4 & 556.7 \\
\hline & with detergent & $158.6^{*}$ & 36.2 \\
\hline \multirow[t]{2}{*}{ Automated washing without pre-washing } & Class B & $135.4^{*}$ & 21.5 \\
\hline & Class A & $119.6^{*}$ & 14.6 \\
\hline \multirow[t]{2}{*}{ Automated washing with pre-washing } & Class B & $72.6^{* \#}$ & 11.5 \\
\hline & Class A & $41.4^{* \#}$ & 7.9 \\
\hline \multirow[t]{2}{*}{ Automated washing with pre-washing after autoclaving } & Class B & $62.4^{*}$ & 9.2 \\
\hline & Class A & $32.7^{*}$ & 6.7 \\
\hline
\end{tabular}

All data are presented as mean \pm SEM for at twenty independent replicates.

${ }^{*} p<0.01$ vs manual washing without detergent; ${ }^{*} p<0.05$ vs automated washing without pre-washing. 
\pm 21.5 for "class B". The use of caging equipment with the above level of contamination is acceptable (Saren et al., 2016 and Smith et al., 2018). After cleaning with the use of universal automatic washers combined with manual prewashing, a two-fold reduction of RLU value to $72.6 \pm 11.5$ for "class B" respectively. This level of ATP detection is the most preferable value for caging equipment. As known from the publications, the hamster's and rabbit's urine contains high levels of inorganic salts forming deposits on the cage surface that are very difficult to remove (Grace et al., 1979, Gunther and Rabinowitz,1980, Nutrient Requirements of Laboratory Animals, 1995). Pre-washing followed by thorough mechanical cleaning and using acidic detergent will significantly improve the overall quality of the cleaning process.

The difference of RLU values (more than five-fold) measured for various microenvironment elements cleaned with the use of automatic washers with/without pre-washing could be explained by the extent of their baseline contamination (in cases where cages are changed after 714 days of use) and the resistance of contaminants (e.g. predominance of bladder stones in hamster cages) to the applied chemical detergents.

After rabbit caging equipment was cleaned in the Atlantis Evo washer ("class A") without pre-washing the mean RLU values amounted to $119.6 \pm 14.6$. When the Atlantis Evo washer in combination with pre-washing was used to clean rabbit caging equipment, the RLU values were similar to those received for the universal washers- $41.4 \pm 7.9$ and $72.6 \pm 11.5$, respectively. However, after using the Atlantis Evo washer the variance of RLU values was smaller. These findings could be associated with a higher temperature of the sanitizing process that enhances the elimination of contaminants.

It is also important to realize that regular washing of polycarbonate and polysulfone items with alkaline agent at a high temperature $\left(>55^{\circ} \mathrm{C}\right)$ will cause accelerated wear of caging equipment. Besides, after being washed cages are damaged by heat sterilization, high-pressure autoclave cycles for prion decontamination, thus reducing their lifespan and therefore decreasing their cost effectiveness.

The concentration of ATP in the microenvironment elements cleaned in automatic washers with pre-washing and after autoclaving was measured (Table 1). The mean RLU values of microenvironment elements automatic washers "class B" and "class A" didn't have significant differences.

To this end, the ABF BIBCh, RAS has developed a specific program combining the pre-washing with alkaline agent at room temperature and the automated washing with acidic detergent at a temperature above $80^{\circ} \mathrm{C}$, followed by sterilization. This washing cycle will ensure the effective removal of mineral portion of the biological contamination (particularly relevant for hamster and rabbit cages) and extend the service life of caging equipment. Also, it will optimize the quality of sanitizing practices.

In summary, based on the above results and the HACCP guidelines, the ABF BIBCh, RAS has developed an assessment scale to monitor sanitizing (Table 2) which defines the following three zones:

- Normal (green)- proper cleanliness of the control point; - Attention is required (yellow)- a higher (vs. cutoff value of the used method) ATP-contamination. It is necessary to verify washing processes in the control points;

- Prohibiting (red)- the control point was not cleaned properly and requires re-sanitization. It is necessary to verify troublefree/proper operation of the washing and disinfecting machines or their hygiene status, as sometimes they can become a source of ATP-contamination (in case of poor cleanliness of their chambers and internal pipelines).

The obtained scale threshold values are based on the multiple ATP tests performed in our facility (as shown in the Table 2, the RLU values did not exceed 119) and the review of the results provided by other researchers (Saren et al., 2016 and Smith et al., 2018). Using the developed scale, the ABF BIBCh, RAS has issued a SOP containing a quarterly schedule to monitor the sanitation of surfaces, materials and equipment. This SOP was included in the AAALAC-approved ABF Animal Care and Use Program.

Analysis of the results obtained with luminometer is a key factor for assessing and continuous improving the sanitization program and, in turn, an approach to promote animal welfare and ensure personnel safety. The extensive experience gained by the ABF BIBCh, RAS in using luminometer and the development of assessment scale for monitoring sanitization through the detection of residual ATPcontamination of micro- and macroenvironment elements provided for the following benefits:

- To establish a verifiable (measurable) monitoring of the quality of sanitization of equipment and surfaces;

- To extend considerably (from 3 to 6 years) the service life of caging equipment used for SPF-animal housing and breeding;

- To spend less money for purchasing expensive detergents and disinfectants;

- To maintain microbiological integrity of the ABF BIBCh, RAS production areas at the appropriate level.

The use of luminometer along with other test systems (Brady temperature indictor strips, disinfection test kit (Browne, Germany), RODAC plates and phenolphthalein test) is a way to estimate and minimize the risks of animal and personnel contamination.

Table 2: Assessment scale for monitoring sanitization of microenvironment elements by residual ATP-contamination (in RLU).

\begin{tabular}{lccc}
\hline & Normal. & Attention! & Do not use! \\
Allowed for use & $0-119$ & Verify the sanitizing process & Re-sanitization is required. \\
\hline Microenvironment & above & 160 & $120-159$ \\
\hline
\end{tabular}




\section{Funding}

The research was supported by the Ministry of Science and Higher Education of the Russian Federation (contract ${ }^{1}$ 07515-2021-1067).

\section{REFERENCES}

Boyce, J.M., Havill, N.L., Dumigan, D.G., Golebiewski, M., Balogun, O. and Rizvani, R. (2009). Monitoring the effectiveness of hospital cleaning practices by use of an adenosine triphosphate bioluminescence assay. Infection Control and Hospital Epidemiology. 30(7): 678-84.

Bruch, M.K. and Smith, F.W. (1968). Improved method for pouring RODAC plates. Applied Microbiology. 16(9): 1427-28.

Dancer, S.J. (2014). Controlling hospital-acquired infection: Focus on the role of the environment and new technologies for decontamination. Clinical Microbiology. 27: 665-690.

Ednie, D.L., Wilson, R.P., Max Lang, C. (1998). Comparison of two sanitation monitoring methods in an animal research facility. Contemporary Topics in Laboratory Animal Science. 37(6): 71-74

Gaylarde, C.C., Bento, F.M., Kelley, J. (1999). Microbial contamination of stored hydrocarbon fuels and its control. Revista de Microbiología. 30: 1-10.

Grace, S.A., Munday, K.A. and Noble, A.R. (1979). Sodium, potassium and water metabolism in the rabbit: the effect of sodium depletion and repletion. Journal of Physiology. 292: 407-20.

Gunther, R.A. and Rabinowitz, L. (1980). Urea and renal concentrating ability in the rabbit. Kidney International. 17: 205-22.

Hacek, D.M., Trick, W.E., Collins, S.M., Noskin, G.A. and Peterson, L.R. (2000). Comparison of the Rodac imprint method to selective enrichment broth for recovery of vancomycinresistant enterococci and drug-resistant Enterobacteriaceae from environmental surfaces. Journal of Clinical Microbiology. 38(12): 4646-8.

Handbook of Hygiene Control in the Food Industry Edited by Lelieveld, H.M., Mostert, M.A., Holah J. Published in North America by CRC Press LLC. (2005). 720 p.

Hart, J.B., French, M.L., Eitzen, H.E. and Ritter, M.A. (1973). RODAC plate-holding device for sampling surfaces during surgery. Applied Microbiology. 26(3): 417-18.

Horn, M.J., Hudson, S.V., Bostrom, L.A. and Cooper, D.M. (2012). Effects of cage density, sanitation frequency and bedding type on animal wellbeing and health and cage environment in mice and rats. Journal of the American Association for Laboratory Animal Science. 51(6): 781-88.

Mulvey, D., Redding, P., Robertson, C., Woodall, C., Kingsmore, P., Bedwell, D. and Dancer, S.J. (2011). Finding a benchmark for monitoring hospital cleanliness. Journal of Hospital Infection. 77(1): 25-30.

Nante, N., Ceriale, E., Messina, G., Lenzi, D. and Manzi, P. (2017). Effectiveness of ATP bioluminescence to assess hospital cleaning: A review. Journal of Preventive Medicine and Hygiene. 58(2): E177-E183.

National Research Council (US) (2011). Committee for the Update of the Guide for the Care and Use of Laboratory Animals. Guide for the Care and Use of Laboratory Animals (The Guide). $8^{\text {th }}$ edition. Washington (DC): National Academies Press (US).

National Research Council (US) (1995). Subcommittee on Laboratory Animal Nutrition. Nutrient Requirements of Laboratory Animals: Fourth Revised Edition, Washington (DC):
National Academies Press (US); (1995). 5, Nutrient Requirements of the Hamster. Available from: https:// www.ncbi.nlm.nih.gov/books/NBK231928.

Rauch, M.E., Graef, H.W., Rozenzhak, S.M., Jones, S.E., Bleckmann, C.A., Kruger, R.L. and Stone, M.O. (2006). Characterization of microbial contamination in United States Air Force aviation fuel tanks. Journal of Industrial Microbiology and Biotechnology. 33(1): 29-36.

Saren, L.E., Hammarberg, L.K., Kastenmayer, R.J. and Hallengren, L.C. (2016). Developing a performance standard for adequate sanitization of wire-bar lids. Journal of the American Association for Laboratory Animal Science. 55(6): 765-68.

Schondelmeyer, C.W., Dillehay, D.L., Webb, S.K., Huerkamp, M.J., Mook, D.M. and Pullium, J.K. (2006). Investigation of appropriate sanitization frequency for rodent caging accessories: evidence supporting less-frequent cleaning. Journal of the American Association for Laboratory Animal Science. 45(6):40-3

Shama, G. and Malik, D. (2013). The uses and abuses of rapid bioluminescence-based ATP assays. International Journal of Hygiene and Environmental Health. 216: 115-125.

Sharapova, T.N., Romanova, E.A., Chernov, A.S., Minakov, A.N., Kazakov, V.A., Kudriaeva, A.A., Belogurov, A.A.Jr, Ivanova, O.K., Gabibov, A.G., Telegin, G.B., Yashin, D.V., Sashchenko, L.P. (2021). Protein PGLYRP1/Tag7 peptides decresases the proinflammatory response in human blood cell and mouse model of diffuse alveolar damage of lung through blockage of the TREM-1 and TNFR1 receptors. Int J Mol Sci. 18; 22(20):11213.

Sherlock, O., O'Connell, N., Creamer, E. and Humphreys, H. (2009). Is it really clean? An evaluation of the efficacy of four methods for determining hospital cleanliness. Journal of Hospital Infection. 72: 140-46.

Smith, B.J., Killoran, K.E., Xu, J.J., Ayers, J.D. and Kendall, L.V. (2018). Extending the use of disposable caging based on results of microbiologic surface testing. Journal of the American Association for Laboratory Animal Science. 57(3): 253-57.

Srinivasan, S., Kaplan, C.N. and Mahadevan, L. (2019). A multiphase theory for spreading microbial swarms and films. Elife. 8: e42697.

Telegin, G.B., Minakov, A.N., Chernov, A.S., Kazakov, V.A., Kalabina, E.A., Manskikh, V.N., Asyutin, D.S., Belogurov, A.A. Jr, Gabibov, A.G., Konovalov, N.A., Spallone, A. (2021). A New Precision Minimally Invasive Method of Glial Scar Simulation in the Rat Spinal Cord Using Cryoapplication Front Surg. 15(8): 607551.

Turner, D.E., Daugherity, E.K., Altier, C. and Maurer, K.J. (2010). Efficacy and limitations of an ATP-based monitoring system. Journal of the American Association for Laboratory Animal Science. 49(2):190-5.

Vilar, M.J., Rodriguez-Otero, J.L., Dieguez, F.J., Sanjuan, M.L., Yus, E. (2008). Application of ATP bioluminescence for evaluation of surface cleanliness of milking equipment. International Journal of Food Microbiology. 125: 357-361.

Whiteley, G.S., Glasbey, T.O. and Fahey, P.P. (2016). A suggested sampling algorithm for use with ATP testing in cleanliness measurement. Infection, Disease and Health. 21(4): 169-75. 